\title{
Avoiding social risk in adolescence
}

Sarah-Jayne Blakemore

UCL Institute of Cognitive Neuroscience

17 Queen Square, London WC1N 3AR

Email: s.blakemore@ucl.ac.uk

Tel: +44 2076791131 


\begin{abstract}
Adolescence is a period of life often characterised by behaviours that, prima facie, are irrational, such as seemingly excessive risk-taking and impulsivity. However, these behaviours can be interpreted as adaptive and rational if one considers that a key developmental goal of this period of life is to mature into an independent adult in the context of a social world that is unstable and changing. It is proposed that, for adolescents, the 'social risk' of being rejected by peers outweighs other potential negative outcomes of decisions, such as threats to one's health or the prospect of getting caught. Furthermore, peer influence in adolescence can lead to prosocial as well as antisocial behaviours.

Neurocognitive mechanisms of peer influence include social reward of being accepted by one's peer group, arousal and increased mentalising, which is associated with development of the social brain network. The findings from cognitive neuroscience and developmental psychology studies fit with recent public health evidence that the opinions of peers are particularly important to adolescents in areas such as school anti-bullying and anti-smoking campaigns.
\end{abstract}

Keywords: adolescence, social cognition, decision-making 


\section{Introduction}

Adolescence is a unique period of development

Adolescence is defined as the period of life between the onset of puberty and adult independence and is a unique period of biological, psychological and social development (Patton et al. 2016). Societal expectations of adolescence differ widely between cultures, and yet, adolescent-typical behaviours can be observed across cultures. A recent study of 5404 people aged 10 to 30 years in 11 different countries showed that, across cultures, sensation-seeking, the desire to experience novel and rewarding stimuli and to take risks, increases throughout the teenage years, is at its highest in the late teens, and then decreases in the twenties. In contrast, self-regulation, the ability to plan and regulate decisions and actions, gradually improves throughout adolescence, eventually stabilising in the midtwenties (Steinberg et al. 2017) (Figure 1). 

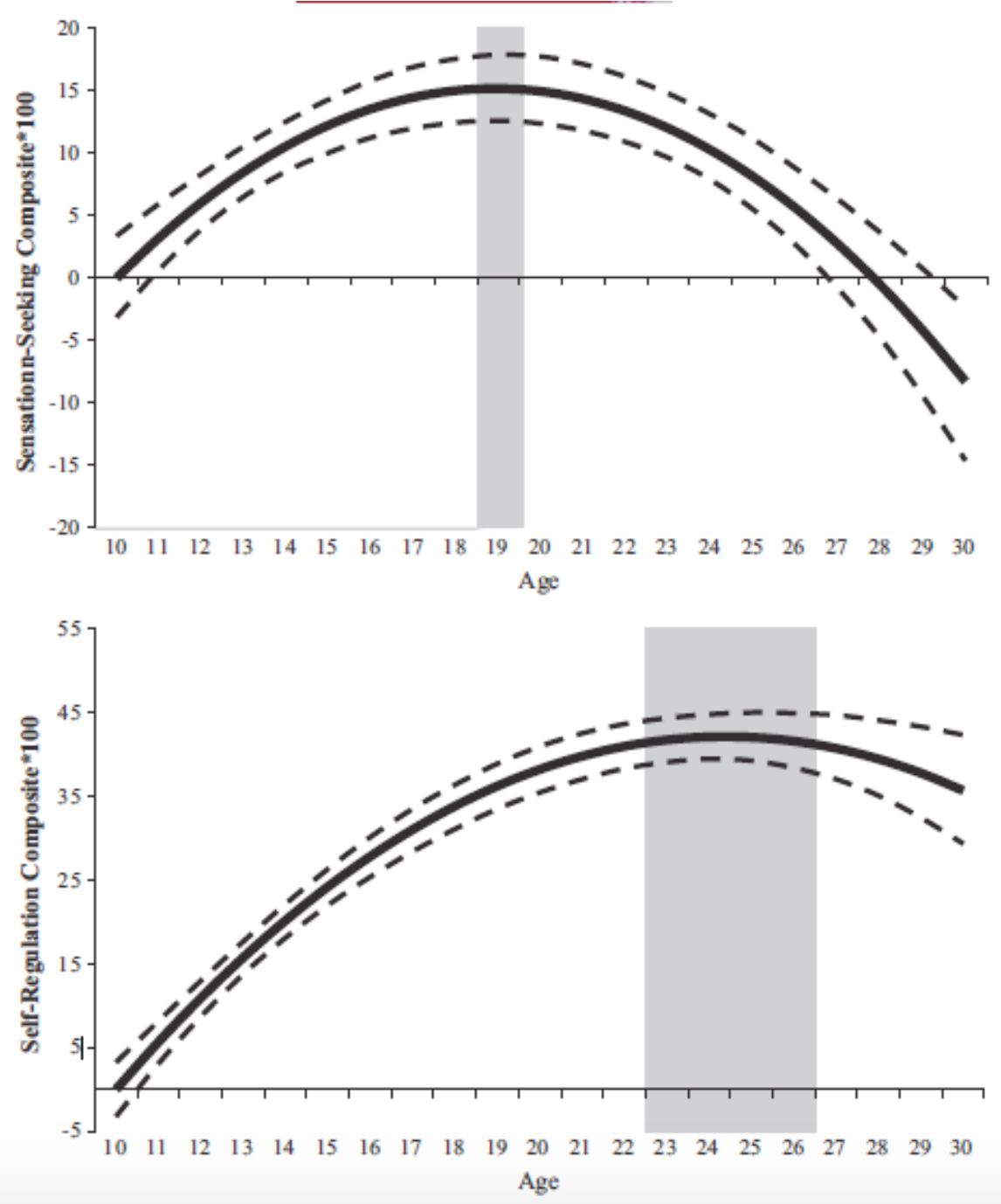

Figure 1: Sensation seeking (top) and self-regulation (bottom) across age in 11 different cultures. Grey shading denotes a plateau/peak, dashed lines indicate 95\% confidence bands. From Steinberg et al. 2017.

Adolescent-typical behaviours can also be observed across species. Mammals undergo a period between puberty and becoming sexually mature adults during which they show increases in exploration, risk-taking, social approach and behaviours and social influence (Adriani et al., 1998; Logue et al. 2014). 
Throughout history, human adolescents have been mocked for their apparently tiresome and hedonistic behaviour. Shakespeare, for example, remarked in The Winter's Tale: "I would there were no age between ten and three-and-twenty, or that youth would sleep out the rest; for there is nothing in the between but getting wenches with child, wronging the ancientry, stealing, fighting".

Underlying the negative stereotype is the assumption that adolescent-typical behaviours such as risk-taking, impulsivity and self-preoccupation are maladaptive and irrational. However, these behaviours can be viewed as adaptive and rational in the context of a key developmental goal of this period of life, that is, to mature into an independent adult (see Romer et al, 2017). During adolescence, young people need to become independent adults by developing a more complete sense of self identity, at the same time as building stronger affiliations with their peer group in the context of a social world that is unstable and changing and when their social networks start out chaotic and only gradually become refined and reciprocated (Burnett-Heyes et al. 2015). Instead of considering adolescence a period of heightened risk-taking per se, a more complete understanding of adolescent-typical behaviours might benefit from a shift in focus to the social context in which risk-taking often occurs.

\section{Do adolescents take more risks?}

Adolescents, as a group, take more risks than children or adults, in domains such as experimentation with illegal substances, risky sexual behaviour, criminal behaviour and driving (Patton et al. 2016). The leading cause of death in young people aged 10-24 years, especially young men, is accidents, which are often caused by risk taking, such as reckless driving (Viner et al. 2011). On this basis, adolescence has been conceptualised as a period in which death is potentially preventable, unlike during other stages of life in which the cause of mortality is primarily health-related (Dahl, 2004). 
However, the picture is more complicated than depicted by the stereotype of the reckless and thrill-seeking adolescent. First, excessive risk-taking resulting in death is fortunately rare, with survival rates of North American high school students being over 99.5\% - most adolescents do not take extreme risks (Willoughby et al. 2014). Even for less extreme risks, there are large individual differences: some individuals are risk-takers, while others are not (Crone et al. 2016). Second, it is important to consider wider contextual factors that enable risk-taking in adolescence. A key contextual factor is the increased freedom permitted by parents and society. Adolescents are given more independence than children, spend more time unsupervised, and are allowed and encouraged to make their own decisions, and this is associated with increased exploration and risk-taking (Borawski et a. 2003).

\section{Peers are a significant determinant of adolescent-typical behaviour}

Another key contextual factor is the increased influence that peers have on adolescents. The amount of time spent with same-sex peers increases between childhood and adolescence, until mid-adolescence (around age 14), when it appears to peak (Lam et al. 2014). What their peers think about them starts to have more influence on adolescents' (13-17 years) evaluation of their social and personal worth (O’Brien \& Bierman, 1998). When adolescents are with peers they are more likely to take risks, such as engaging in reckless behaviour and experimenting with drugs, alcohol and cigarettes, compared to when they are alone (Reniers et al. 2016). Lab experiments have shown that adolescents (aged 13-24 years) are more likely to take driving risks when with friends, compared with when alone; in contrast, adults' ( 25 years and over) driving risks are unaffected by peers (Gardner \& Steinberg, 2005). This is mirrored by findings from data from car accidents, which indicate that the risk of accidents for young drivers is heightened when they have a passenger in the car (Chen et al. 2000). Adolescents' substance use behaviour is also influenced by peers. Studies in Hong Kong, for example, have shown that having friends who smoke or drink alcohol is the biggest predictor 
of adolescent smoking and drinking (Yuen Loke \& Mak, 2013). A longitudinal study involving adolescents (aged 10-15 years at the start of the study) in California, showed that perceived peer cannabis use predicts onset and extent of the adolescent's own cannabis use over the next three years; a similar relationship was found for alcohol use (D'Amico \& McCarthy, 2006).

Many studies of peer influence in adolescence have included only one age group, so often comparisons across age cannot be made. A study that included a large age range demonstrated that young adolescents' views about risk are especially influenced by other teenagers' views (Knoll et al. 2015). In this study, 563 participants aged between 8 and 59 years were asked to rate the riskiness of everyday situations and were then presented with risk ratings of the same situations from other people, either teenagers or adults (these provided ratings were in fact fictitious). Participants were then asked to rate the riskiness of the situations again. All age groups were influenced by other people's opinion but, while children and adults were more influenced by the opinions of adults, young adolescents (aged 12-14 years) changed their ratings more towards the ratings of teenagers than towards the ratings of adults (Figure 2; this result was partially replicated in a new group of 590 participants in Knoll et al. 2017). 


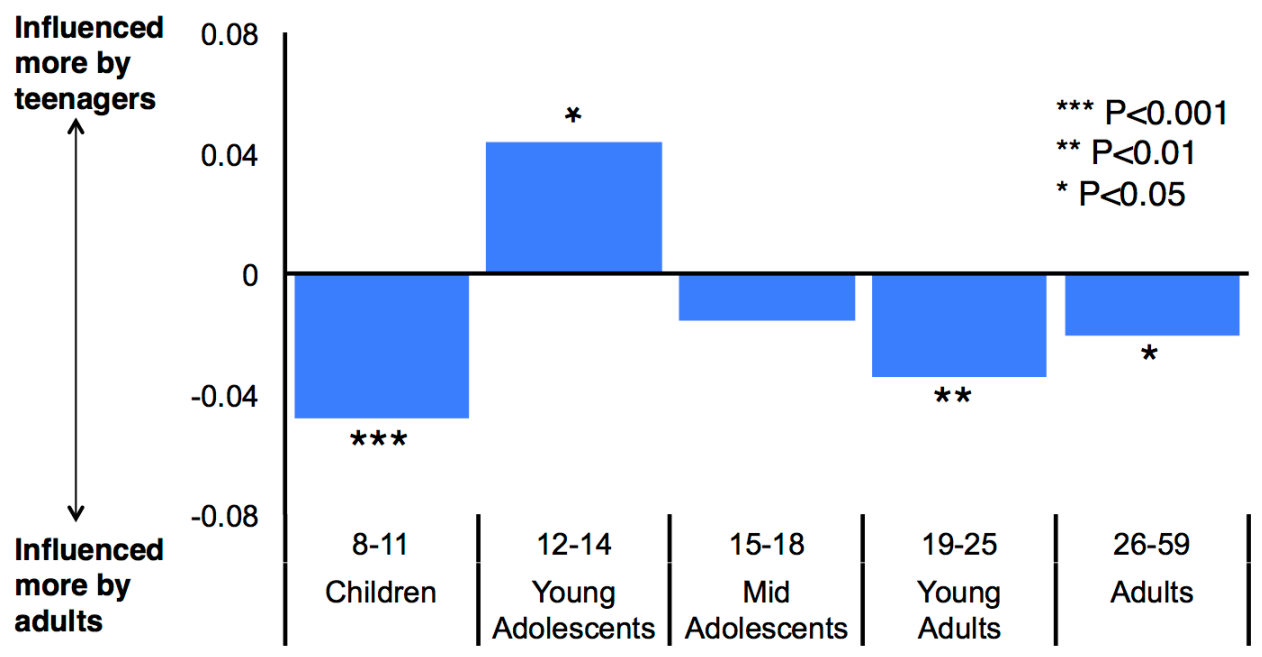

Figure 2: Social influence on risk perception across age. Unlike other age groups, young adolescents are more influenced by the views of teenagers than by those of adults. From Knoll et al. 2015.

Peer influence can also have positive effects: a friend who discourages a young person from engaging in a particular risky behaviour can reduce the tendency of the young person engaging in that activity (Maxwell, 2002). Adolescents aged 12-15 years are more likely to volunteer to help others in their community if they believe other students in their school volunteer (Choukas-Bradley et al., 2015). A study that employed a public goods game showed that 12-16 year olds gave a more generous allocation of coins to their group after they saw peers approve such behaviour (Van Hoorn et al. 2016). Adolescents sometimes engage in risky decisions with the intention of helping other individuals, such as sticking up for a friend who is being bullied even though this risks becoming a victim of the bullies - this has been labelled 'prosocial risk taking' by Telzer and colleagues (Do et al. 2017). Thus, peer influence in adolescence can lead to prosocial as well as antisocial behaviours.

There are still many remaining questions about peer influence in adolescence. As is clear from the studies described above, the precise ages at which risk-taking and peer influence 
peak are highly variable between studies and this is partly because there are large individual differences. What underlies individual differences in peer influence? Another important question concerns why adolescents are particularly susceptible to peer influence, and that is what I turn to next.

\section{Avoiding the risk of social rejection}

It has been proposed that adolescents are particularly susceptible to peer influence because they are hypersensitive to social exclusion (Blakemore \& Mills, 2014). In a commonly employed social exclusion paradigm, Cyberball, in which participants play an online game of catch with two other (fictitious) players, adolescents' mood is lowered more than adults' after being excluded by the other players (Sebastian et al. 2010). Individual differences in susceptibility to peer influence are pronounced, and one study showed that adolescents with low resistance to peer influence take more driving risks (in a driving video game) following social exclusion in Cyberball than adolescents with high resistance to peer influence (Peake et al. 2013).

Overall though, adolescents appear to be hypersensitive to social exclusion, and this might lead to the risk of social rejection - or 'social risk' - being particularly pronounced for adolescents. We have proposed that, in adolescence, social risk is weighted more strongly in decisions than other factors such as potential health or legal risks (Blakemore \& Mills, 2014). In this context, going along with peers in order to avoid social risk, even if it means taking health and legal risks, might be seen as the rational choice because it reduces the possibility of social exclusion.

What is classified as a social risk is an open question, but the minimal prediction would be that any decision or action that might lead to peer exclusion would be considered a social risk. Social risk could be broader still and include anything that might lead to a reduction in one's 
position in the social hierarchy, or even mild embarrassment or loss of face. It has been proposed that avoiding social risk in adolescence is important for mental health in adolescence. Hypersensitivity to the threat of social risk, or chronic social rejection, might be associated with mental illnesses such as depression (Allen \& Badcock, 2003), a condition that usually has its onset in early to mid-adolescence (Joinson et al. 2017). A study with over 1200 adolescents and young adults (aged 14-24 years), showed that self-reported friendship quality in adolescence predicts mental health resilience, defined as better than expected psycho-social functioning (lower psychiatric symptoms and higher mental wellbeing) one year later (Van Harmelen et al. 2017). This study did not measure social risk-taking, and it assessed self-reported friendship quality (dyadic relationships) rather than feelings of peer acceptance or peer influence, and the links that can be made between this and social-risk avoidance are limited. However, the results suggest there is adaptive advantage in high perceived friendship quality and putative links with social-risk avoidance could be explored in future studies.

\section{Neurocognitive mechanisms of peer influence}

There are multiple, non-mutually exclusive, possible neurocognitive mechanisms that lead to the presence of peers influencing adolescents' decisions and actions. One possibility is that increased sensitivity to social reward underlies peer influence. fMRI studies have demonstrated increased activity in the ventral striatum and orbitofrontal cortex, regions involved in reward processing, in adolescents relative to adults when they take simulated driving risks in the imagined presence of peers (Chein et al. 2011) (note though that these regions are involved in many processes other than reward).

A second possibility is that the real or imagined presence of peers results in changes in arousal levels, and this in turn might affect behaviour. In an fMRI study, on trials in which participants thought they were being observed while having their brain scanned, and even in 
anticipation of being observed, adolescents reported higher levels of embarrassment and showed higher skin conductance - a measure of arousal - than did children or adults (Somerville et al. 2013). In addition, thinking they were being watched, and anticipating being watched, was associated with greater activity in the dorsomedial prefrontal cortex (dmPFC), a region within the social brain, in adolescents compared with children. In the presence of peers, or when anticipating being evaluated by peers, increased fear of social evaluation might lead adolescents to think more about how peers judge their behaviour. In this way, peers might lead to increased mentalising, the attribution of mental states and emotions to other people - a third possible mechanism underlying peer influence.

Mentalising ability improves between late childhood and early adulthood (Dumontheil et al. 2010). In addition, the 'social brain' network, including the dmPFC, anterior temporal cortex, posterior superior temporal sulcus and temporo-parietal junction, develops both in terms of grey matter and cortical thickness (Mills et al. 2012) and activity in mentalising tasks (Blakemore, 2008) during adolescence. This might equip adolescents with increasingly sophisticated abilities to understand other people's minds, at a stage when they are motivated to become affiliated with their peer group and to understand what their peers think of them. Further evidence for the involvement of the social brain comes from the fMRI study by Peake and colleagues, which demonstrated that the activity in the right temporo-parietal junction during risky decisions on a driving game mediated the relationship between resistance to peer influence and risk-taking after social exclusion in Cyberball (Peake et al 2013).

\section{Is adolescence a sensitive period of social brain development?}

It is possible that adolescence represents a late sensitive period of brain development, during which the brain is particularly susceptible to certain environmental input, including social stimuli. The heightened effect of peer influence was demonstrated in a rodent study that showed that male adolescent mice (P28-P30) consume more alcohol when with other 
mice than when alone, whereas alcohol consumption in male adult mice (P84-P86) remained the same whether alone or with other mice (Logue et al. 2014). In male rats, social stress, such as social isolation, during adolescence has larger effects on adult social and sexual behaviour (McCormick et al. 2013) and brain structure (Whitaker et al. 2013), compared with the same social stress imposed earlier or later in development. Most rodent studies have been conducted in males and results are less consistent for females (Burke et al. 2017). Overall however, the studies suggest that the presence of other rodents during adolescence is critical for healthy social and neural development.

As deprivation experiments would not be ethical to carry out in humans, another way to study sensitive periods is to assess age differences in the ability to acquire information. If a neural network is undergoing a period of heightened plasticity during a particular age range, individuals in that age range might be particularly adept at learning social or nonsocial information processed by that network (see Knoll et al. 2016). Future studies could investigate age differences in the acquisition of social information to test the hypothesis that the acquisition of complex social information about peers is at its highest in adolescence.

\section{Implications for public health}

Public health advertising aimed at young people's predilection for risky behaviours tends to focus on the health risks of these behaviours. However, the research reviewed in this paper, as well as research in the public health domain (e.g. Dishion \& Dodge, 2005), suggests that focusing on social norms and peer expectations might have more impact on adolescent behaviour. This was supported by a public health study that looked at the influence of social norms on bullying behaviour and conflict in schools (Paluck et al. 2016). Fifty-six middle schools (with children aged 11-16 years) in the USA were included, with half the schools being assigned at random to an anti-bullying programme. A number of students in each year 
participated in the programme and were encouraged to lead grassroots anti-bullying campaigns in their schools and become the public face of opposition to bullying. Compared with control schools, in which no special anti-bullying programmes had been introduced, reports of student conflict at the schools that had received the student-led anti-bullying programme were reduced by $30 \%$. Furthermore, when the anti-bullying campaign was led by more popular students, it had a greater positive effect on behaviour.

A similar result was reported in a randomised control trial in which 12-13-year-old students in British schools who were rated by their classmates as influential were trained as 'peer supporters' to try to encourage their peers not to smoke (Campbell et al. 2008). Over 10,000 12-13-year-olds took part in this study and the results showed a significant reduction in smoking over a two-year period following the peer support intervention compared with the control group of schools in which no special programme was put in place.

These studies reveal the power of peer influence in changing social norms of acceptable behaviour. Adolescent susceptibility to peer influence and the motivation to avoid social risk can be beneficial and should be harnessed by public health campaigns aimed at young people.

\section{Acknowledgements and endnotes}

*Address correspondence to Sarah-Jayne Blakemore, UCL Institute of Cognitive Neuroscience, 17 Queen Square, London WC1N 3AR, UK

The author is supported by the Royal Society, the Wellcome Trust and the Jacobs Foundation. Many thanks to colleagues who commented on previous versions of this paper, including C. Frith, U. Frith, L. Foulkes, A-L. Goddings, T. Dalgleish, C. Sebastian, L. Magis-Weinberg, AL van Harmelen, D. Fuhrmann and K Mills. 


\section{References}

Adriani, W., Chiarotti, F., Laviola, G. (1998). Elevated novelty seeking and peculiar Damphetamine sensitization in periadolescent mice compared to adult mice. Behav Neuroscienwce. 112, 1152-1166.

Allen, N. B., Badcock, P. B. (2003). The social risk hypothesis of depressed mood: evolutionary, psychosocial, and neurobiological perspectives. Psychol Bull. 129 (6), 887-913 Blakemore, S-J. (2008). The social brain in adolescence. Nature Reviews Neuroscience 9 , $267-277$

Blakemore, S-J., Mills, K. L. (2014). Is Adolescence a Sensitive Period for Sociocultural Processing? Annu. Rev. Psychol. 65, 187-207

Borawski, E. A., Ievers-Landis, C. E., Lovegreen, L. D., Trapl, E. S. (2003). Parental monitoring, negotiated unsupervised time, and parental trust: the role of perceived parenting practices in adolescent health risk behaviors. $J$ Adolesc Health. 33 (2), 60-70 Burke, A. R., McCormick, C. M. Pellis, S. M., Lukkes J. L., (2017). Impact of adolescent social experiences on behavior and neural circuits implicated in mental illnesses. Neurosci Biobehav Rev. pii: S0149-7634(16)30050-1. doi: 10.1016/j.neubiorev.2017.01.018. [Epub ahead of print]

Burnett-Heyes, S., Jih, Y.R., Block, P., Hiu, C. F., Holmes, E. A., Lau, J. Y. (2015).

Relationship Reciprocation Modulates Resource Allocation in Adolescent Social Networks: Developmental Effects. Child Dev. 86 (5), 1489-506

Campbell R1, Starkey F, Holliday J, Audrey S, Bloor M, Parry-Langdon N, Hughes R, Moore L. (2008). An informal school-based peer-led intervention for smoking prevention in adolescence (ASSIST): a cluster randomised trial. Lancet 371(9624), 1595-602. Chein, J., Albert, D., O'Brien, L., Uckert, K. Steinberg, L. (2011). Peers increase adolescent risk taking by enhancing activity in the brain's reward circuitry. Dev Sci., 14 (2), F1-10 Chen, L., Baker, S. P., Braver, E. R. Li, G. (2000). Carrying passengers as a risk factor for crashes fatal to 16- and 17-year old drivers. JAMA, 283 (12),1578-82 
Choukas-Bradley, S., Giletta, M., Cohen, G. L., Prinstein, M. J. (2015). Peer Influence, Peer Status, and Prosocial Behavior: An Experimental Investigation of Peer Socialization of Adolescents' Intentions to Volunteer. $J$ Youth Adolesc. 44 (12), 2197-210

Crone, E.A., van Duijvenvoorde, A. C., Peper, J. S. (2016). Neural contributions to risktaking in adolescence developmental changes and individual differences. $J$ Child Psychol Psychiatry. 57 (3), 353-68

D'Amico, E. J., McCarthy, D. M. (2006). Escalation and initiation of younger adolescents' substance use: the impact of perceived peer use. J Adolesc Health 39 (4), 481-7

Dahl, R.E. (2004). Adolescent brain development: a period of vulnerabilities and opportunities. Ann N Y Acad Sci., 1021, 1-22

Dishion, TJ, Dodge, KA. (2005). Peer Contagion in Interventions for Children and Adolescents: Moving Towards an Understanding of the Ecology and Dynamics of Change. $J$ Abnorm Child Psychol. 33(3), 395-400.

Do, KT, Moreira, JFG, Telzer, EH. (2017). But is helping you worth the risk? Defining Prosocial Risk Taking in adolescence. Devel Cogn Neurosci 25, 260-271.

Dumontheil, I., Apperly, I.A., Blakemore, S-J. (2010). Online usage of theory of mind continues to develop in late adolescence. Developmental Science 13, 331-338

Gardner, M., Steinberg, L. (2005). Peer influence on risk taking, risk preference, and risky decision making in adolescence and adulthood: An experimental study. Dev Psychol. 41, $625-635$

Joinson, C., Kounali, D., Lewis, G. (2017). Family socioeconomic position in early life and onset of depressive symptoms and depression: a prospective cohort study. Soc Psychiatry Psychiatr Epidemiol. 52 (1), 95-103

Knoll, L. J., Fuhrmann, D., Sakhardande, A., Stamp, F., Speekenbrink, M., Blakemore, S-J. (2016). A window of opportunity for cognitive training in adolescence. Psychological Science. 27(12), 1620-1631 
Knoll, L. J., Magis-Weinberg, L., Speekenbrink, M., Blakemore, S-J. (2015). Social influence on risk perception during adolescence. Psychol Sci. 26 (5), 583-92

Knoll, L.J., Leung, J.T., Foulkes, L. \& Blakemore, S-J. (2017) Age-related differences in social influence on risk perception depend on the direction of influence. $J$ Adolesc. 60, 53-63. Lam, C. B., McHale, S. M., Crouter, A. C. (2014). Time With Peers From Middle Childhood to Late Adolescence: Developmental Course and Adjustment Correlates. Child Dev. 85 (4), $1677-1693$

Logue, S., Chein, J., Gould, T., Holliday, E., Steinberg, L. (2014). Adolescent Mice, Unlike Adults, Consume More Alcohol in the Presence of Peers than Alone. Dev Science. 17(1), 7985.

Maxwell, K. A. (2002). Friends: The role of peer influence across adolescent risk behaviors. J. Youth Adolesc. 32, 267-277

McCormick, C. M., Green, M. R. Cameron, N. M., Nixon, F., Levy, M.J., Clark, R. A. (2013). Deficits in male sexual behavior in adulthood after social instability stress in adolescence in rats. Horm. Behav. 63, 5-12

Mills, K. L., Lalonde, F., Clasen, L. S., Giedd, J. N., Blakemore, S-J. (2012). Developmental changes in the structure of the social brain in late childhood and adolescence. Social Cognitive and Affective Neuroscience 9, 123-131

O’Brien, S. F., Bierman, K. L. (1988). Conceptions and perceived influence of peer groups: interviews with preadolescents and adolescents. Child Dev. 59 (5), 1360-5

Paluck, E. L., Shepherd, H., Aronow, P. M. (2016). Changing climates of conflict: A social network experiment in 56 schools. Proc Natl Acad Sci U S A. 113(3), 566-71

Patton, G.C, Sawyer, S.M, Santelli, J.S., Ross, D.A., Afifi, R. et al. (2016). Our future: a Lancet commission on adolescent health and wellbeing. Lancet. 387, 2423-78.

Peake, S. J., Dishion, T. J., Stormshak, E. A., Moore, W. E., Pfeifer, J. H. (2013). Risk-taking and social exclusion in adolescence: neural mechanisms underlying peer influences on decision-making. Neuroimage 82, 23-34 
Reniers, R. L., Beavan, A., Keogan, L., Furneaux, A. Mayhew, A., Wood, S. J. (2016). Is it all in the reward? Peers influence risk-taking behaviour in young adulthood. Br J Psychol.

108 (2), doi: 10.1111/bjop.12195

Romer D, Reyna VF, Satterthwaite TD (2017). Beyond stereotypes of adolescent risk taking: Placing the adolescent brain in developmental context. Dev Cogn Neurosci. 27, 19-34.

Sebastian, C., Viding, E., Williams, K., Blakemore, S-J. (2010). Social brain development and the affective consequences of ostracism in adolescence. Brain \& Cognition 72, 134-145 Somerville, L. H. (2013). Special issue on the teenage brain: Sensitivity to social evaluation. Curr Dir Psychol Sci. 22 (2), 121-127

Somerville, L. H., Jones, R. M., Ruberry, E. J., Dyke, J. P., Glover, G., Casey, B. J. (2013). The medial prefrontal cortex and the emergence of self-conscious emotion in adolescence. Psychol Sci. 24 (8), 1554-62

Steinberg, L., Icenogle, G., Shulman, E.P., Breiner, K., Chein, J. et al. (2017). Around the world, adolescence is a time of heightened sensation seeking and immature self-regulation. Dev Sci. doi: $10.1111 /$ desc. 12532

Van Harmelen, A. L., Kievit, R. A., Ioannidis, K., Jones, P. B., Bullmore, E. et al., Adolescent friendships predict later resilient functioning across psychosocial domains in a healthy community cohort. Psychological Medicine, in press (available at $\underline{\text { http://discovery.ucl.ac.uk/1544179/). }}$

Van Hoorn, J., Van Dijk, E., Güroğlu, B., Crone, E. A. (2016). Neural Correlates of Prosocial Peer Influence on Public Goods Game Donations During Adolescence. Soc Cogn Affect Neurosci 11(6), 923-933

Viner, R.M., Coffey, C., Mathers, C., Bloem, P., Costello, A. et al. (2011). 50-year mortality trends in children and young people: a study of 50 low-income, middle-income, and highincome countries. Lancet 377, 1162-74

Whitaker, L. R., Degoulet, M., Morikawa, H. (2013). Social deprivation enhances VTA synaptic plasticity and drug-induced contextual learning. Neuron $\mathbf{7 7}, 335-45$ 
Willoughby, T., Tavernier, R., Hamza, C., Adachi, P. J., Good, M. (2014). The triadic systems model perspective and adolescent risk taking. Brain Cogn. 89, 114-5

Yuen Loke, A., Mak, Y-W (2013). Family Process and Peer Influences on Substance Use by Adolescents. Int J Environ Res Public Health 10 (9), 3868-3885 


\section{Recommended reading}

Crone EA, Dahl RE. (2012). Understanding adolescence as a period of social-affective engagement and goal flexibility. Nat Rev Neurosci. 13(9), 636-650. This review paper evaluates the evidence that social-affective processing is a critical component of adolescent neurocognitive and behavioural development.

Pattwell SS, Duhoux S, Hartley CA, Johnson DC, Jing D, Elliott MD, Ruberry EJ, Powers A, Mehta N, Yang RR, Soliman F, Glatt CE, Casey BJ, Ninan I, Lee FS. (2012). Altered fear learning across development in both mouse and human. Proc Natl Acad Sci U S A. 109(40), 16318-16323. This study investigated the development of fear extinction learning in mice and humans, and demonstrated attenuated extinction learning during adolescence in both populations and altered synaptic plasticity in the mouse brain, underlining the importance of combining methodologies and species for a more complete understanding of the mechanisms of neurocognitive development.

Tamnes CK, Herting MM, Goddings AL, Meuwese R, Blakemore S-J, Dahl RE, Güroğlu B, Raznahan A, Sowell ER, Crone EA, Mills KL. (2017). Development of the cerebral cortex across adolescence: A multisample study of interrelated longitudinal changes in cortical volume, surface area and thickness. $J$ Neurosci. 37 (12), 3402-3412. This study analysed longitudinal structural imaging data from 391 individuals aged between eight and 30 years from four developmental cohorts from three countries, and showed remarkable consistency in developmental trajectories of the brain across samples.

Blakemore, S-J., Mills, K. L. (2014). Is Adolescence a Sensitive Period for Sociocultural Processing? Annu. Rev. Psychol. 65, 187-207. This review discusses social brain development and the propensity for peer influence in adolescence in more detail than in the current paper. 